\title{
Processing and analysis of the measured alignment errors for RHIC*
}

\author{
F. Pilat, M. Hemmer, V. Ptitsin, S. Tepikian, and D. Trbojevic \\ Brookhaven National Laboratory, Upton, NY, 11973, USA
}

\begin{abstract}
All elements of the Relativistic Heavy Ion Collider (RHIC) have been installed in ideal survey locations, which are defined as the optimum locations of the fiducials with respect to the positions generated by the design. The alignment process included the presurvey of all elements which could affect the beams. During this procedure a special attention was paid to the precise determination of the quadrupole centers as well as the roll angles of the quadrupoles and dipoles. After installation the machine has been surveyed and the resulting as-built measured position of the fiducials have been stored and structured in the survey database. We describe how the alignment errors, inferred by comparison of ideal and as-built data, have been processed and analyzed by including them in the RHIC modeling software. The RHIC model, which also includes individual measured errors for all magnets in the machine and is automatically generated from databases, allows the study of the impact of the measured alignment errors on the machine.
\end{abstract}

\section{MEASUREMENT TECHNIQUES}

The centers of the RHIC quadrupoles were determined in two ways: by ferrofluidic colloidal cell and by antenna measurements. The colloidal cell [1] uses a collimated light passing through two polarizers at the opposite sides of the magnet. This light cannot be seen with the telescope if there is no magnetic field around the cell. In the presence of field, small particles in the colloidal solution orient themselves along the magnetic field lines producing the field picture at the telescope. The antenna [2] [3] is a special coil harmonic with several windings to measure harmonic and feed down terms. Fiducials are placed on the antenna to locate the magnetic centers relative to the magnet fiducials.

Information about the survey instruments and data collection can be found in [4].

\section{FINDING THE OPTIMAL MAGNET POSITIONS}

A set of programs have been developed to ensure that all RHIC magnets are installed as close as possible to their design position, in the "ideal" position, [5]. Mechanical and optical survey data are collected, structured in a relational database (SYBASE) and analyzed. For all magnets, the mechanical roll angle measurements were very well correlated with the field roll measurements. The cold mass fiducials, welded near the ends of the laminations, are accessible during optical survey in the production phase and during magnet positioning in the tunnel. The position of the cold mass fiducials are located with respect to the cryostat fiducials and to the horizontal gravitational plane. We will describe in more detail what has been done for the main magnet systems, dipoles and quadrupoles, with particular emphasis on the Interaction Region quadrupoles.

Arc dipoles have 4 cold mass fiducials. The dipole magnet centers were determined by a combination of the optical survey and mechanical dial gauge indicator measurements. The roll of the dipoles was controlled by a combination of the rotating coil measurements and the optical survey measurements. The field angle of the dipoles is determined by the rotating coil measurements with respect to the gravitational plane. The optical survey measurements were performed at room temperature with only a few magnets measured at $4 \mathrm{~K}$. The warm to cold correlation for the field angle has a standard deviation of $0.20 \mathrm{mrad}$. The syatematic error as well as the reproducibility of the measurement is estimated to be $\pm 0.2 \mathrm{mrad}$.

Arc quadrupoles have also 4 fiducials and their magnetic centers were determined by the best linear fit over a few positions along the length of the magnets. This was used to define the beam axis vector. The systematic error in the location of the quadrupole magnetic center along the magnets, measured by two methods (cell and antenna), was estimated to be within $\pm 60 \mu \mathrm{m}$.

Special attention was paid to the properties and alignment of the Interaction Region (IR) magnets, the strong focusing quadrupoles (triplets), dipoles, and harmonic correctors since the IRs dominate the beam dynamics at storage. There are three packages of multi-layer correction magnets between the triplet magnets [3]. Two are directly attached to $\mathrm{Q} 3$ while one is attached to the $\mathrm{Q} 2$ magnet. Q1, the shorter cold mass, has 4 fiducials, Q2 and Q3 respectively have 10 and 12 fiducials since they are longer and at a more critical position. The quanties relevant to the alignment and positioning of the IR assemblies are summarized in Table 1. Mechanical measurements such as the sag and

Table 1: Measured quantities in IR assemblies

\begin{tabular}{lll}
\hline \hline Mechanical & Correction package & Survey data \\
Sag & FA between layers & QC along length \\
Roll angle & FA to quad & FA along length \\
CM fiducials & FC along length & CM fiducials \\
CM length & FC each layer & CR fiducials \\
& & BPM offset \\
\hline
\end{tabular}

* Work performed under the auspices of the U.S. Department of Energy 
roll angle along the length,the cold mass (CM) fiducials with respect to the mechanical center, and the CM length from end plate to end plate were taken during production. The second column lists quantities relevant to the correction package assembly, the field angle (FA) between layers, the residual FA relative to the quadrupole FA, the position of the field centers (FC) along the length, the position of FC of each layer. The third colums lists survey and ficld data, the quadrupole center (QC) and FA along the length, the CM fiducial and cryostat (CR) fiducials in the local coordinate system. Table 2 shows the average FA and standard deviation for the IR triplet and special dipoles. Table 3 shows the standard deviations of the magnetic centers for the same magnet assemblies, radial, along the magnet axis and vertical respectively. The positions of the two beam

Table 2: Measured Field Angle for RHIC IR assemblies

\begin{tabular}{lll}
\hline \hline Magnet & FA mean [rad] & FA sigma [rad] \\
CQ1 & -0.001101 & 0.001227 \\
CQ2 & -0.000553 & 0.001013 \\
CQ3 & -0.000726 & 0.001219 \\
D0+ & -0.000385 & 0.000931 \\
D0- & -0.000476 & 0.000924 \\
DX & -0.001236 & 0.000768 \\
\hline
\end{tabular}

Table 3: Measured magnetic centers for RHIC IR assemblies

\begin{tabular}{llll}
\hline \hline Magnet & $\mathbf{x}$ [in] & $\mathbf{y}$ [in] & $\mathbf{z}$ [in] \\
CQ1 & 0.0178 & 0.1086 & 0.0092 \\
CQ2 & 0.0965 & 0.0783 & 0.0182 \\
CQ3 & 0.0929 & 0.0710 & 0.0196 \\
D0+ & 0.3278 & 0.0288 & 0.0106 \\
D0- & 0.3165 & 0.0285 & 0.0148 \\
DX & 0.1554 & 0.1101 & 0.0285 \\
\hline \hline
\end{tabular}

position monitors (BPM) were also measured with respect to the quadrupole centers by a separate RF antenna system. The accuracy of the BPM position with respect to the outside fiducials is estimated to be $\pm 0.13 \mathrm{~mm}$. The survey and alignment of the high beta elements were performed by using eight theodolites at the same time, electronically connected to a portable computer. Adjustments of the magnet positions are performed until the difference between the required and established positions in readings of at least four instruments are less than $\pm 25 \mu \mathrm{m}$. Other details about the surveying procedure were presented elsewhere [4].

\section{ANALYSIS OF RHIC MISALIGNMENT}

Magnets were installed in their ideal positions, as determined by the ideal fiducials. Once installed, all magnets have been surveyed and the real position of the fiducials have been measured (as-built fiducials). For each magnet we calculate the difference (delta) between the ideal and the as-built values at each fiducial, and we define as magnet misalignment the average over the deltas. The goal of the analysis is to evaluate the impact of the misalignment on the machine performance, notably the closed orbit, to establish if the dipole corrector system is able to compensate for it and to recommend resurveying of specific magnets if necessary. We pursued two methods of analysis: the first directly correlates the errors to the strengths of neighboring corrctors as a first check. The second includes the same misalignment data in the detailed model of RHIC allowing the first turn and the correction of the closed orbit to be realistically simulated. The analysis of the vertical alignment will be described in the following, the horizontal analysis is work in progress.

\subsection{Correlation of errors to correctors}

Figure 1 shows the vertical alignment for both Yellow and Blue RHIC rings. These main cause of errors is ground settlement in the tunnel. The correlation between Blue and Yellow is very good. Although the data exibit long wavelength oscillations, the real source of the beam orbit perturbation are short range local irregularities or spikes corresponding to big relative misalignments of individual quadrupoles. In order to quantatively characterize the

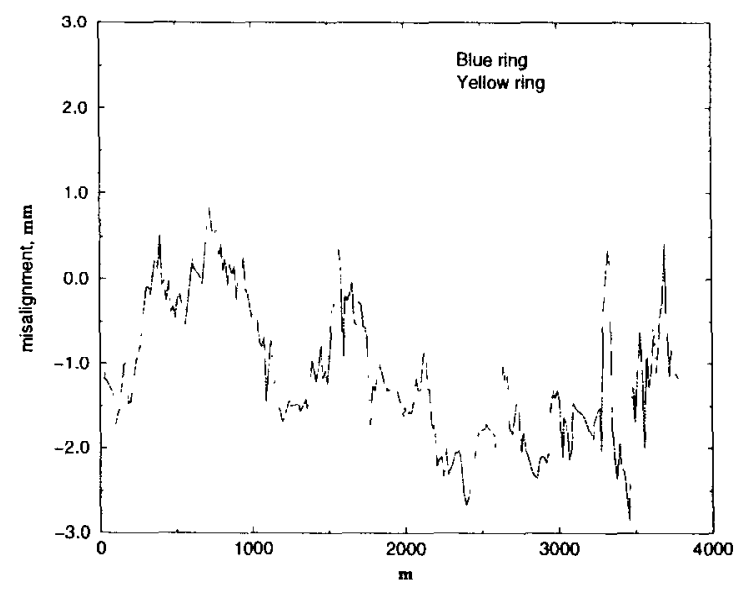

Figure 1: Vertical misalignment data for quadrupoles

strength of the orbit perturbation produced by the errors we used the concept of effective corrector strength. That is, the value of the corrector strength, (adjacent to the quadrupole) which is required to locally correct the beam orbit. This involves the relative misalignments of 3 neighboring quadrupoles. Figures 2 and 3 show the effective corrector strengths expressed in terms of the orbit kick angle for vertically misaligned quadrupoles. The dashed lines mark the effective region $( \pm 90 \mu \mathrm{rad})$ inside which we would like to limit the corrector strength at top energy. The required strengths in the region between $3000 \mathrm{~m}$ and 4000 $\mathrm{m}$ is notably worse than in other areas. Quadrupoles here 


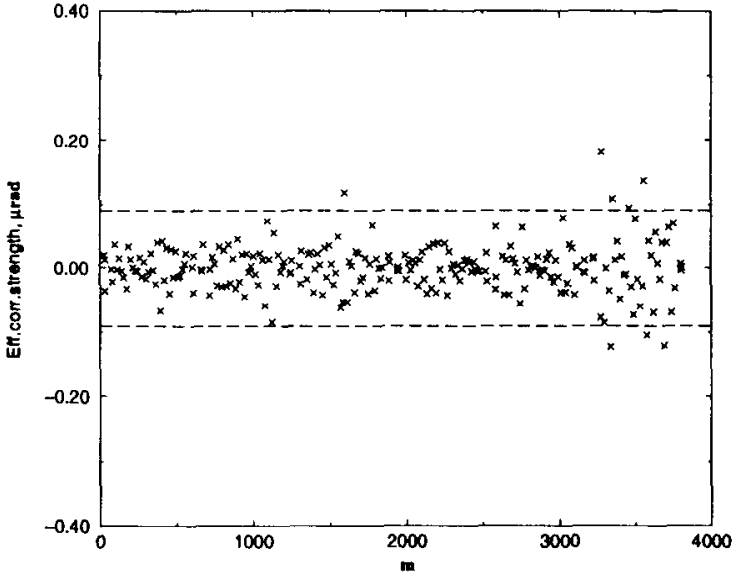

Figure 2: Effective corrector strengths for the Blue Ring

were the first to be installed, and on the basis of the present analysis the magnets in this sector have been realigned. A

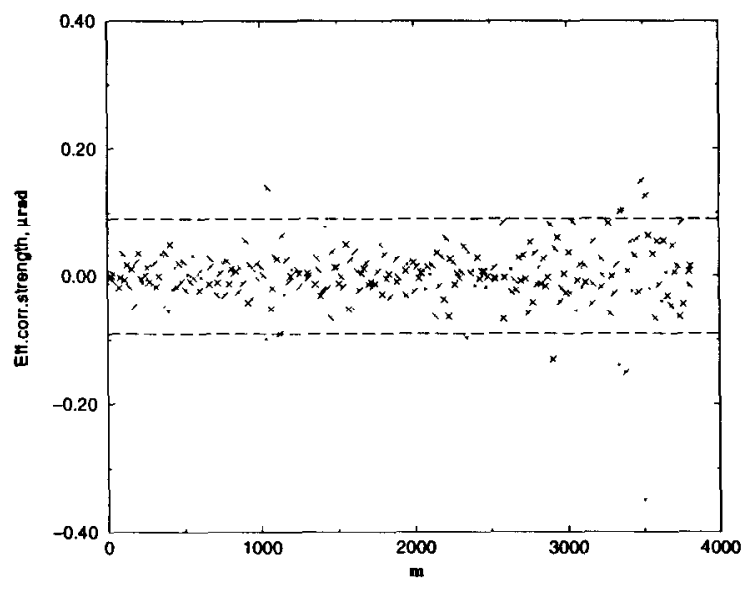

Figure 3: Effective corrector strengths for the Yellow ring

good example is the point in Figure 3 that requires about $-350 \mu \mathrm{rad}$ corrector strength, far beyond the available corrector strength at the top energy. This quadrupole, $O 04 Q 20$ needed to be realigned by about $3 \mathrm{~mm}$. It is remarkable that the same realignment value was calculated on the basis of beam loss monitor data taken with beam during the RHIC sextant test [7].

\subsection{Modeling of RHIC}

A software model has been developed for RHIC that uses UAL [8], a modular environment which provides an accelerator object model and physics libraries interfaced via Perl Scripts, and SXF [9], an ascii external machine representation which includes errors together with lattice information. The model includes individual measurement field harmon- ics assigned to all measured magnets in the ring. The RHIC model is automatically generated from the lattice, magnet and survey databases by a collection of stored procedures, $\mathrm{C}++$ code, and Perl scripts. We extended the model to include the measured misalignments by assigning the data to the magnets via a filter that reads, manipulates and writes out a RHIC SXF file. The resulting machine has been simulated by TEAPOT++ with particular focus on the first turn and closed orbit correction. The study confirmed that the measured alignment errors in RHIC can be handled by the closed orbit correction system. Figure 4 is an example of the vertical beam orbit during the first turn before and after correction (by the sliding bump method system that will be used for real first turn steering in RHIC [6]).

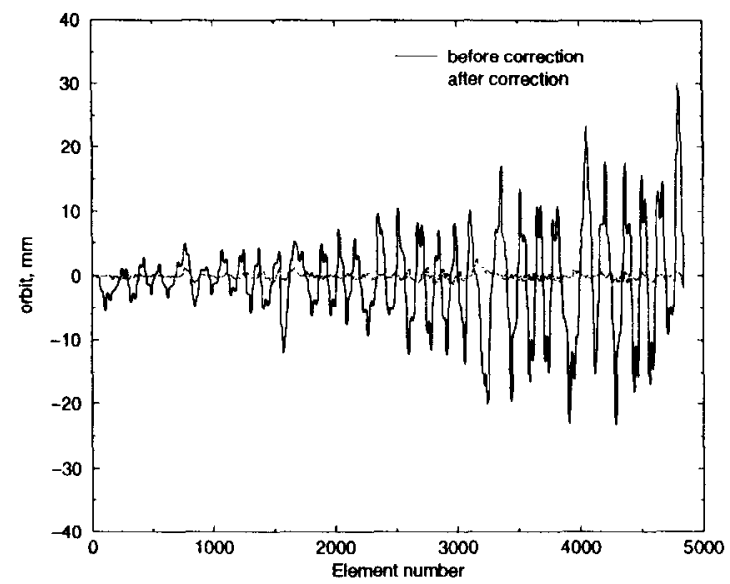

Figure 4: Correction of the vertical orbit (first turn).

\section{CONCLUSIONS}

The positioning of all magnets in RHIC has been optimized and all magnets have been eventually surveyed. The impact of the measured vertical misalignment on the closed orbit was found, after resurveying of a few quadrupoles, in within the capability of the dipole correction system.

\section{REFERENCES}

[1] M.A. Goldman, et. al., PAC93, Washington, pp.2916-2918.

[2] D. Trbojevic et.al., PAC95, Dallas, pp.2099-2101.

[3] D. Trbojevic et al., PAC97, Vancouver, pp.3651-3654.

[4] M. Hemmer, "RHIC Electronic Data Collection and Survey and Alignment Database", BNL Internal Document, August 1993.

[5] W. Mackay, S. Peggs, RHIC/AP/12, Nov. 1993.

[6] W.Fischer et al.,"The RHIC Injection System”, PAC99. New York City.

[7] P.Thompson, private communication

[8] N.Malitsky, R.Talman, AIP 391, 1996

[9] H.Grote, et.al., RHIC/AP 155 